\title{
Desigualdad del ingreso y desarrollo del sistema financiero un enfoque multidimensional
}

\author{
Tomás Gómez Rodríguez ${ }^{1}$ - Universidad Autónoma del Estado de Hidalgo, México \\ Humberto Ríos Bolívar - Instituto Politécnico Nacional, México \\ Adriana Zambrano Reyes - Universidad Autónoma del Estado de Hidalgo, México
}

Este trabajo explora la relación entre el sistema financiero y la desigualdad del ingreso. Se utilizó el método de Mínimos Cuadrados Ordinarios como estimación base y el método de Mínimos Cuadrados Generalizados Estimados con efectos aleatorios de dos vías como prueba de robustez. Los resultados para el sector bancario indican que profundidad, eficiencia y estructura de mercado exhiben evidencia a favor de la hipótesis de un aumento de la desigualdad del ingreso. De igual manera, se encuentra evidencia de una relación positiva entre menores niveles de competencia y la desigualdad del ingreso. Se recomienda de acuerdo a los resultados garantizar condiciones equitativas en la utilización de los servicios financieros. La limitante de este estudio es que no se consideró la dimensión acceso para el sector bancario. La aportación original es utilizar cuatro medidas diferentes de desigualdad del ingreso. La dimensión profundidad tanto para el sector bancario como para los mercados financieros, tiene el efecto de aumentar la desigualdad del ingreso. Mientras que la dimensión estabilidad del sector bancario y la dimensión eficiencia de los mercados financieros tienen un efecto redistributivo.

Clasificación JEL: G10, C33, D3.

Palabras clave: Sistema Financiero, panel de datos, desigualdad del ingreso.

\section{Income Inequality and Financial System Development a Multidimensional Approach}

This paper explores the relationship between the financial system and income inequality. The Ordinary Least Squares method was used as the base estimation and the Generalized Least Squares Estimated method with two-way random effects as a robustness test. The results for the banking sector indicate that depth, efficiency and market structure exhibit evidence in favor of the hypothesis of an increase in income inequality. Similarly, there is evidence of a positive relationship between lower levels of competition and income inequality. According to the results, it is recommended to guarantee a level playing field in the use of financial services. The main limitation of this study is that the access dimension was not considered for the banking sector. The original contribution of this research is to use four different measures of income inequality. The depth dimension for both the banking sector and financial markets has the effect of increasing income inequality. While the stability dimension of the banking sector and the efficiency dimension of financial markets have a redistributive effect. JEL Clasification: G10, C33, D3.

Keywords: Financial system, data panel, Income inequality.

${ }^{1}$ Autor de correspondencia: Tomás Gómez Rodríguez. Dirección: Carretera Apan-Calpulalpan Km.8, Col.Chimalpa, C.P. 43920 Apan, Hgo. Teléfono: 017717172000 extensión 5806. Correo electrónico: tomas_gomez@uaeh.edu.mx.

*Sin fuente de financiamiento para el desarrollo de la investigación. 


\section{Introducción}

Los recientes cambios registrados en la desigualdad del ingreso y en el desarrollo del sistema financiero a nivel internacional han atraído el interés de investigadores y legisladores. El aumento de la desigualdad del ingreso es uno de los desafíos más importantes para los legisladores tanto en países desarrollados como en desarrollo. Este aumento de la desigualdad del ingreso implica más injusticia económica, inestabilidad social y desigualdad en las oportunidades de desarrollo. Estos problemas a su vez pueden ocasionar inestabilidad política (Nollert, 1995, Boix, 2008 y Solt, 2015), desigualdad del poder político (Goodin y Dryzek, 1980 y Solt, 2008) y crisis financieras (Stiglitz, 2009 y Bjørnskov, 2015). Por lo tanto, la desigualdad del ingreso daña el desarrollo económico y social (Chiu y Lee, 2019).

Como consecuencia se han realizado numerosas investigaciones que estudian el origen y las consecuencias socio-económicas de dicho fenómeno. Especialmente existe una literatura naciente que explora la relación entre el desarrollo del sistema financiero y la desigualdad del ingreso muestra de esto son los trabajos de: Zhang y Naceur (2019), Meniago y Asongu (2018), Chiu y Lee (2019), Hsieh, Chen y Lin (2019), Altunbaş y Thornton (2019) y Thornton y di Tommaso (2019). Esta rama de la literatura considera que cuando el sector financiero genera mayor crecimiento económico, los individuos de un país pueden alcanzar un mayor ingreso y por tanto alcanzar una distribución del ingreso más igualitaria. Este aumento en el ingreso de los individuos es posible debido a que el sistema financiero tiene la capacidad de colocar los recursos financieros en su mejor uso. Tanto el sector bancario como los mercados financieros son los mejores posicionados para desempeñar esta tarea, gracias a su capacidad de movilizar los recursos financieros de toda una economía (Meniago y Asongu, 2018). En contraste en un escenario donde el acceso a los servicios financieros se da de acuerdo al ingreso de los individuos, el desarrollo del sistema financiero puede significar un crecimiento de la desigualdad del ingreso. Por tanto, el sector financiero repercute en las oportunidades y la riqueza de las personas. Lo anterior sugiere la existencia de un vínculo entre el desarrollo del sistema financiero y la distribución del ingreso (Demirgüç-Kunt y Levine, 2009).

Por otro lado, los cambios ocurridos en las condiciones competitivas en el sector bancario a nivel internacional en tiempos recientes han generado inquietud acerca del posible impacto del poder de mercado en el sector bancario sobre las variables macroeconómicas (Hsieh, Chen y Lin, 2019). Especialmente el interés está en el posible impacto que la estructura de mercado del sector bancario puede tener sobre la distribución del ingreso. Ya que en la medida que el poder de mercado en la banca determine la disponibilidad de crédito y la inversión para las empresas medianas y pequeñas, este será un factor determinante en el nivel de empleo y la desigualdad del ingreso. Esto se debe a que dichas empresas son más sensibles a los problemas de información y son más dependientes del financiamiento bancario que las empresas grandes. Sobre todo, es importante considerar que las empresas medianas y pequeñas son una fuente relevante de empleo de acuerdo a Hsieh, Chen y Lin (2019). La literatura existente sobre el nexo entre la estructura de mercado del sector bancario y la desigualdad del ingreso es incipiente, la mayor parte de la investigación que se ha realizado hasta ahora se ha centrado en los nexos entre estructura de mercado y estabilidad (Allen y Gale, 2004), así como en el nexo entre estructura de mercado y eficiencia. Algunos trabajos que 
tratan estos temas son los de: Boyd y de Nicoló (2005), Schaeck, Cihak y Wolfe (2009), Rice y Strahan (2010) y Beck, de Jonghe y Schepens (2013).

La literatura existente sobre el nexo entre el desarrollo del sistema financiero y la desigualdad del ingreso se puede dividir en dos hipótesis. La primera hipótesis propone que el desarrollo del sistema financiero tiene un efecto positivo sobre la desigualdad del ingreso. Mientras que la segunda hipótesis establece que el desarrollo del sistema financiero tiene una relación negativa sobre la desigualdad del ingreso (Chiu y Lee, 2019).

Por otro lado, las principales hipótesis en cuanto a la relación entre la estructura de mercado del sector bancario y la desigualdad del ingreso son dos la primera implica que el poder de mercado puede crear mayor desigualdad del ingreso (hipótesis de poder de mercado), mientras que la segunda sugiere que mayor poder de mercado puede disminuir la desigualdad (hipótesis de información) (Hsieh, Chen y Lin, 2019). De acuerdo a la primera hipótesis, mayor poder de mercado puede resultar en una menor oferta de préstamos y tasas más altas. Mientras que mayor competencia permite un mayor acceso al financiamiento lo que significaría una reducción de la desigualdad del ingreso. La hipótesis de la información argumenta que el poder de mercado resulta en una mayor inversión en la relación que tienen los bancos con sus clientes, lo que a su vez reduce las asimetrías de información y los costos de agencia. Esto trae como resultado una mejora en el acceso al financiamiento por parte de los potenciales solicitantes de crédito. Sobre todo, de aquellos solicitantes con información opaca o que suponen mayor riesgo como las empresas pequeñas o aquellas con problemas financieros. Por tanto, en presencia de mayor competencia los bancos podrían no aprovechar su ventaja en información y fallar en establecer relaciones cercanas. Por tanto, mayor competencia significa una profundización del crédito para algunas empresas y falta de crédito para otras, aumentando así la desigualdad del ingreso (Hsieh, Chen y Lin, 2019).

Por lo tanto, este estudio tiene tres propósitos. El primero es analizar la existencia de una relación positiva entre el desarrollo del sector bancario y la desigualdad del ingreso. El segundo es evaluar si las características de los mercados financieros tienen el efecto de aumentar la desigualdad del ingreso. Por último, se evalúa el impacto positivo del crecimiento de la estructura de mercado del sector bancario sobre la desigualdad del ingreso.

Para probar lo anterior se emplean dos muestras la primera se centra en el sector bancario y la segunda se enfoca en los mercados financieros. La primera muestra está compuesta por 75 países y la segunda muestra abarca 57 países. El marco temporal para ambas muestras abarca del año 1999 al 2012. Los países que componen ambas muestras se pueden consultar en el apéndice en las tablas 1 y 2. Siguiendo el trabajo de Zhang y Naceur (2019) se propone evaluar las siguientes dimensiones del desarrollo financiero para el sector bancario, estas son: profundidad, eficiencia, estabilidad y competencia; en contraste con el trabajo de Zhang y Naceur (2019) se considera una dimensión adicional la cual es la estructura de mercado. Mientras que para los mercados financieros se analizan: el acceso la profundidad, la eficiencia, la rentabilidad y la estabilidad. A diferencia de los trabajos de Zhang y Naceur (2019) y Meniago y Asongu (2018) donde se emplean paneles de datos no balanceados, en la presente investigación se emplean paneles de datos balanceados. Por otro lado, las variables utilizadas en los trabajos de Zhang y Naceur (2019) y Meniago y Asongu (2018) están en niveles, mientras que en la presente investigación las variables se presentan en tasas de crecimiento. Asimismo, para analizar la desigualdad se emplean las siguientes medidas: el índice de Gini, el índice de Atkinson, la proporción Media a Mediana, la proporción Palma y el índice de Theil. 
Se emplea el método de Mínimos Cuadrados Ordinarios (MCO) para la estimación base y el método de Mínimos Cuadrados Generalizados Estimados con efectos aleatorios de dos vías como prueba de robustez.

El resto de esta investigación tiene la siguiente estructura. En la sección 2 se realiza una revisión de la literatura, la cual consiste en un estudio de los principales trabajos sobre el tema. La sección 3 contiene la descripción de las variables a utilizar y además se registran las estadísticas básicas. La sección 4 comprende los métodos de estimación. En la sección 5 se presentan los resultados de la estimación econométrica, así como los resultados de la prueba de robustez. La última sección contiene las conclusiones.

\section{Revisión de la literatura}

Existen dos teorías principales en el estudio del nexo entre desarrollo financiero y desigualdad del ingreso. La primera de estas teorías propone que el desarrollo del sistema financiero tiene un efecto positivo sobre la desigualdad del ingreso, es decir el desarrollo del sistema financiero beneficia más a los ricos que a los pobres lo que amplía la desigualdad del ingreso. La segunda teoría establece que el desarrollo del sistema financiero tiene una relación negativa sobre la desigualdad del ingreso. Esta teoría sugiere que mayor desarrollo financiero puede ayudar a las personas con ingresos bajos a tener acceso al financiamiento externo.

La primera teoría se basa en el modelo de Banerjee y Newman (1993) el cual está construido bajo el supuesto de que las finanzas pueden proveer oportunidades a los emprendedores. Sin embargo, las imperfecciones financieras como altos costos de transacción y el cumplimiento de los contratos impide que las personas de bajos recursos puedan invertir y convertirse en emprendedores. Esto se debe a que las personas de bajos recursos muchas veces no tienen historial creditico o el colateral necesario para recibir financiamiento de las instituciones financieras. Esto a su vez ocasiona que personas con proyectos que pueden obtener ganancias altas no los realicen por falta de crédito y terminen trabajando para mejores empleadores, pero con un ingreso menor al que podrían obtener. El modelo propone que mientras que los emprendedores puedan tener acceso al capital sin importar las posibles restricciones, se traduce en una reducción de la desigualdad del ingreso (Meniago y Asongu, 2018).

La segunda teoría tiene su fundamento en el trabajo de Galor y Zeira (1993) el cual se basa en la afirmación de que las disparidades en el ingreso evitan que se realice una colocación eficiente de los recursos cuando los mercados financieros son imperfectos, esta situación impide que las personas de bajos recursos inviertan en capital humano y físico. Y se centra en el argumento de que los individuos tienen las mismas capacidades y habilidades potenciales, pero diferentes dotaciones de recursos debido a la riqueza heredada. Como consecuencia de la información imperfecta y los altos costos de transacción. Las personas de bajos recursos enfrentan limitaciones de crédito y por lo tanto invertirán menos en capital humano en comparación con las personas de altos recursos. En el modelo los individuos que reciben una herencia invertirán en capital humano, entonces la riqueza futura de los hogares depende de la herencia recibida. Los hogares de las personas con altos recursos que invierten en educación obtienen más habilidades, lo que les permite obtener un ingreso mayor y dejar a sus descendientes una mayor herencia. Mientras que los hogares pobres no pueden invertir 
en educación lo que reduce su preparación, esta falta de habilidades se traduce en ingresos bajos y en la imposibilidad de dejar una herencia a las próximas generaciones. Aun si las personas de bajos ingresos tuvieran la posibilidad de invertir en capital humano, las imperfecciones de los mercados financieros evitarían que lo hicieran. Por tanto, en el largo plazo la distribución del ingreso está determinada por la inversión en capital humano, lo que a su vez depende de la riqueza heredada (Meniago y Asongu, 2018).

Existe un desacuerdo entre estas hipótesis y este mismo desacuerdo también existe en los resultados de la investigación empírica. Por un lado, se encuentran los estudios que aportan evidencia a favor de la hipótesis de la reducción de la desigualdad entre estos están: Galor y Zeira (1993), Deininger y Squire (1998); Ravallion (2004): White y Anderson (2001), Dollar y Kraay (2004), Clarke, Xu y Zou (2006), Beck, Demirgüç-Kunt y Levine (2007), Johansson y Wang (2014), von Ehrlich y Seidel (2015), Hamori y Hashinguchi (2012), Agnello, Mallick y Sousa (2012), Kunieda, Okada y Shibata (2013), Zhang y Naceur (2019), Altunbaş y Thornton (2019) y Thornton y di Tommaso (2019).

El trabajo de Kunieda, Okada y Shibata (2013) registra los resultados de tres estimaciones diferentes: la primera es un estudio de sección cruzada con una muestra de 119 países, la segunda estimación es un panel de datos con efectos fijos con una muestra de 120 países y la tercera estimación utiliza el método generalizado de momentos con una muestra de 17 países. Los resultados del estudio muestran que el desarrollo financiero reduce la desigualdad en los países con economías cerradas. Con una muestra de 143 países en un período de 1961 a 2011, Zhang y Naceur (2019) emplean un enfoque de variables instrumentales para estimar la relación entre diferentes dimensiones del desarrollo financiero y desigualdad del ingreso. Las conclusiones del estudio son que la profundización del sistema financiero tiene una relación negativa con la desigualdad del ingreso, mientras que la liberalización financiera aumenta la desigualdad del ingreso. Empleando el método de regresión en cuantiles para una muestra de 121 países Altunbaş y Thornton (2019), encuentra que el efecto del desarrollo financiero varía de acuerdo al nivel de ingreso de un país. En los países con un ingreso medio superior el efecto del desarrollo financiero es promover la igualdad, mientras que en los países de ingreso alto y bajo el desarrollo financiero juega un papel importante en el aumento de la desigualdad. Thornton y di Tommaso (2019) se examina la relación de largo plazo entre desarrollo financiero y desigualdad del ingreso. La muestra que emplean consiste en 119 países para el período de 1980-2015. Haciendo uso de diferentes técnicas de cointegración encuentran que el desarrollo del sistema financiero reduce la desigualdad del ingreso.

Por otro lado, existen trabajos que refutan la hipótesis de que el desarrollo financiero tenga un efecto reductor en la desigualdad del ingreso, estos trabajos son los de: Banerjee y Newman (1993), Rajan y Zingales (2003), Gimet y Lagoarde-Segot (2011), Sehrawat y Giri (2015), Jauch y Watzka (2015), Denk y Cournede (2015), Seven y Coskun (2016) y de Haan y Sturm (2016).

Los resultados del trabajo de Jauch y Watzka (2015) muestran que cuando existe mayor desarrollo del sistema financiero aumenta la desigualdad del ingreso. La estimación se realiza empleando un panel de datos no balanceado de 138 países con efectos individuales y efectos temporales, para el período 1960 a 2008. Empleando una muestra de 33 países de la OCDE en un período de 1970 a 2011. Seven y Coskun (2016) hacen uso del método generalizado de momentos, con una muestra de 45 países emergentes en el período 1987-2011 los datos se estructuran en seis promedios de 4 años. Ellos encuentran que, aunque el crecimiento económico se ve afectado 
REMEF (The Mexican Journal of Economics and Finance)

Desigualdad del ingreso y desarrollo del sistema financiero un enfoque multidimensional

positivamente por el desarrollo del sistema financiero, no se encuentra un efecto reductor de la desigualdad del ingreso por parte del desarrollo financiero. Usando un panel de datos con efectos fijos y una muestra de 121 países que cubre el período 1975-2005 de Haan y Sturm (2016), encuentran que todas las variables financieras utilizadas en el estudio incrementan la desigualdad del ingreso.

En cuanto a la estructura de mercado, las teorías presentan conclusiones en conflicto. La teoría del poder del mercado argumenta que un mayor nivel de competencia amplía el acceso al financiamiento. Acceso fácil a los servicios financieros significa mayor oferta de crédito y menores tasas de interés y por tanto debería reducir la desigualdad del ingreso. Mientras que la hipótesis de la información postula que, en presencia de competencia, los bancos no pueden capitalizar su ventaja en la información y por tanto no pueden establecer relaciones cercanas con sus clientes (Hsieh, Chen y Lin, 2019). Esta situación disminuye el acceso para empresas pequeñas y profundiza el acceso al crédito por parte de las empresas grandes, lo que provoca un aumento de la desigualdad del ingreso. En cuanto a la evidencia empírica el trabajo de Hsieh, Chen y Lin (2019) es el primero en analizar la relación entre estructura de mercado y desigualdad del ingreso. Su estudio encuentra que menor competencia en el sector bancario tiene un efecto positivo sobre la desigualdad del ingreso, estos efectos son mayores durante los períodos de crisis, al igual que para países con alto ingreso o en países con mejores instituciones políticas.

\section{Datos}

Se utilizaron dos muestras diferentes: la primera muestra contiene datos de diferentes dimensiones del sector bancario para 75 países y la segunda muestra se enfoca en los mercados financieros y se conforma por 57 países. Ambas muestras abarcan el período 1999-2012 y conforman dos paneles de datos balanceados. La elección de países y del marco temporal se vio influenciada por la disponibilidad de los datos. Se utiliza la desigualdad del ingreso como variable dependiente, la cual se mide a través de cinco medidas diferentes: el índice de Gini, el índice de Atkinson, el índice de Theil, la proporción de Palma y la proporción media a mediana. Los datos provienen del Proyecto Global de Consumo e Ingreso elaborado por Lahoti, Jayadev y Reddy (2016). Esta base a su vez está basada en fuentes secundarias como: el Estudio de Ingreso de Luxemburgo, La Base Mundial de Desigualdad del Ingreso y la base del Banco Mundial Povcalnet entre otras. El índice de Gini (Des. Gini) tiene una escala de uno a cero, cuando el valor es cero implica que todos en la sociedad reciben el mismo ingreso. Por otro lado, si el valor es uno indica que una persona recibe todo el ingreso. El índice de Atkinson (Des. Atkinson) mide el porcentaje del ingreso total que una sociedad debe renunciar para que exista una participación más igualitaria del ingreso entre los individuos de una sociedad. La proporción Palma (Des. Palma) considera el decil más alto de la distribución del ingreso con respecto al cuarto decil del fondo de la distribución. La principal ventaja tanto del índice de Atkinson como de la proporción Palma en la medición de la desigualdad del ingreso es que capturan las colas de la desigualdad entre los más pobres y los más ricos, a diferencia del índice de Gini que considera la totalidad de la distribución del ingreso (Cobham Schlögl y Summer, 2016). Por otro lado, el índice de Theil (Des. Palma) mide la desigualdad del ingreso a través del cálculo de la entropía. El término entropía significa desorden, pero cuando se aplica a una distribución del ingreso 
significa desviarse de la igualdad perfecta. Para este índice se pueden observar valores de ente 0 y 1 , si el valor es 1 entonces el ingreso está en su peor distribución. Por último, para complementar las medidas de desigualdad utilizamos la proporción media a mediana (Des. $m 2 m$ ), la cual tiene la ventaja de ser una medida clara y simple, que no ha sido utilizada en la literatura debido a que los datos no estaban disponibles (Birdsall y Meyer 2015). En general, valores altos para todas estas medidas significan mayor desigualdad del ingreso, mientras que valores más bajos significan una distribución del ingreso más igualitaria.

Tabla 1. Variables dependientes.

\begin{tabular}{|c|c|c|c|c|c|}
\hline Tipo & Dimensión & Variable & Abreviatura & Fuente & $\begin{array}{l}\text { Unidad de } \\
\text { medida }\end{array}$ \\
\hline $\begin{array}{l}\text { Desigualdad } \\
\text { del ingreso }\end{array}$ & $\begin{array}{l}\text { Macro- } \\
\text { económica }\end{array}$ & Índice de Gini & Des.Gini & $\begin{array}{l}\text { Proyecto } \\
\text { Global de } \\
\text { Consumo } \\
\text { e Ingreso }\end{array}$ & $\begin{array}{c}\text { Tasa de } \\
\text { crecimiento } \\
(\%)\end{array}$ \\
\hline $\begin{array}{l}\text { Desigualdad } \\
\text { del ingreso }\end{array}$ & $\begin{array}{l}\text { Macro- } \\
\text { económica }\end{array}$ & $\begin{array}{l}\text { Índice de } \\
\text { Atkinson }\end{array}$ & Des.Atkinson & $\begin{array}{l}\text { Proyecto } \\
\text { Global de } \\
\text { Consumo } \\
\text { e Ingreso }\end{array}$ & $\begin{array}{c}\text { Tasa de } \\
\text { crecimiento } \\
(\%)\end{array}$ \\
\hline $\begin{array}{l}\text { Desigualdad } \\
\text { del ingreso }\end{array}$ & $\begin{array}{l}\text { Macro- } \\
\text { económica }\end{array}$ & Índice de Theil & Des.Theil & $\begin{array}{l}\text { Proyecto } \\
\text { Global de } \\
\text { Consumo } \\
\text { e Ingreso }\end{array}$ & $\begin{array}{c}\text { Tasa de } \\
\text { crecimiento } \\
(\%)\end{array}$ \\
\hline $\begin{array}{l}\text { Desigualdad } \\
\text { del ingreso }\end{array}$ & $\begin{array}{l}\text { Macro- } \\
\text { económica }\end{array}$ & $\begin{array}{l}\text { Proporción } \\
\text { Palma }\end{array}$ & Des.Palma & $\begin{array}{l}\text { Proyecto } \\
\text { Global de } \\
\text { Consumo } \\
\text { e Ingreso }\end{array}$ & $\begin{array}{c}\text { Tasa de } \\
\text { crecimiento } \\
(\%)\end{array}$ \\
\hline $\begin{array}{l}\text { Desigualdad } \\
\text { del ingreso }\end{array}$ & $\begin{array}{l}\text { Macro- } \\
\text { económica }\end{array}$ & $\begin{array}{l}\text { Proporción } \\
\text { Media a } \\
\text { Mediana }\end{array}$ & Des. $m 2 m$ & $\begin{array}{l}\text { Proyecto } \\
\text { Global de } \\
\text { Consumo } \\
\text { e Ingreso }\end{array}$ & $\begin{array}{c}\text { Tasa de } \\
\text { crecimiento } \\
(\%)\end{array}$ \\
\hline
\end{tabular}

Fuente: Elaboración propia.

En cuanto a las variables independientes tenemos dos conjuntos. El primer conjunto se centra en el sector bancario y evalúa las siguientes dimensiones: profundidad, eficiencia, estabilidad y estructura de mercado. La dimensión profundidad se analiza a través de la variable crédito domestico al sector privado (P.Credit), valores más altos para este variable sugieren un sector bancario más profundo. La dimensión eficiencia se mide a través de dos variables: el rendimiento bancario sobre los activos (E.return) y el margen de interés neto (E.net_interest), valores altos para el margen de interés sugieren mayor eficiencia, así mismo, valores más altos para la variable rendimiento bancario apuntan a una mayor eficiencia.

La dimensión estabilidad considera el Z-Score (Estab.Z_score) y la proporción crédito bancario a depósitos bancarios (Estab.cred_dep), valores más altos para la variable Z-score implican una menor probabilidad de bancarrota, mientras que valores más altos para la proporción crédito bancario a depósitos bancarios sugieren una posible falta de liquidez en un futuro. 
Por último, para la dimensión estructura de mercado se utilizan tanto el índice de concentración de los cinco bancos más importantes (Estru.Conc) como el índice de Lerner (Estru.Lerner) si el índice de concentración es alto esto supone un bajo nivel de competencia. Si el índice de Lerner es cero se piensa que el mercado está en competencia perfecta, si el índice de Lerner es 1 el mercado está en monopolio y entre 0 y 1 el mercado muestra competencia monopolística.

Tabla 2. Variables del sector bancario

\begin{tabular}{|c|c|c|c|c|c|}
\hline Tipo & Dimensión & Variable & Abreviatura & Fuente & $\begin{array}{l}\text { Unidad de } \\
\text { medida }\end{array}$ \\
\hline $\begin{array}{l}\text { Sector } \\
\text { Bancario }\end{array}$ & Profundidad & $\begin{array}{l}\text { Crédito } \\
\text { domestico al } \\
\text { sector } \\
\text { privado/PIB }\end{array}$ & P.Credit & $\begin{array}{l}\text { Base de } \\
\text { Datos de } \\
\text { Estructura } \\
\text { Financiera }\end{array}$ & $\begin{array}{c}\text { Tasa de } \\
\text { crecimiento } \\
\text { (\%) }\end{array}$ \\
\hline $\begin{array}{l}\text { Sector } \\
\text { Bancario }\end{array}$ & Eficiencia & $\begin{array}{l}\text { Margen de } \\
\text { interés neto }\end{array}$ & E.net_interest & $\begin{array}{l}\text { Base de } \\
\text { Datos de } \\
\text { Estructura } \\
\text { Financiera }\end{array}$ & $\begin{array}{c}\text { Tasa de } \\
\text { crecimiento } \\
(\%)\end{array}$ \\
\hline $\begin{array}{l}\text { Sector } \\
\text { Bancario }\end{array}$ & Eficiencia & $\begin{array}{l}\text { Rendimiento } \\
\text { sobre los activos }\end{array}$ & E.return & $\begin{array}{l}\text { Base de } \\
\text { Datos de } \\
\text { Estructura } \\
\text { Financiera }\end{array}$ & $\begin{array}{c}\text { Tasa de } \\
\text { crecimiento } \\
(\%)\end{array}$ \\
\hline $\begin{array}{l}\text { Sector } \\
\text { Bancario }\end{array}$ & Estabilidad & Z-score & Estab.Z_score & $\begin{array}{l}\text { Datos de } \\
\text { Estructura } \\
\text { Financiera }\end{array}$ & $\begin{array}{c}\text { Tasa de } \\
\text { crecimiento } \\
\text { (\%) }\end{array}$ \\
\hline $\begin{array}{l}\text { Sector } \\
\text { Bancario }\end{array}$ & Estabilidad & Crédito/depósito & Estab.cred_dep & $\begin{array}{l}\text { Datos de } \\
\text { Estructura } \\
\text { Financiera }\end{array}$ & $\begin{array}{c}\text { Tasa de } \\
\text { crecimiento } \\
\text { (\%) }\end{array}$ \\
\hline $\begin{array}{l}\text { Sector } \\
\text { Bancario }\end{array}$ & $\begin{array}{l}\text { Estructura de } \\
\text { mercado }\end{array}$ & $\begin{array}{l}\text { Índice de } \\
\text { concentración }\end{array}$ & Estru.Conc & $\begin{array}{l}\text { Datos de } \\
\text { Estructura } \\
\text { Financiera }\end{array}$ & $\begin{array}{c}\text { Tasa de } \\
\text { crecimiento } \\
(\%)\end{array}$ \\
\hline $\begin{array}{l}\text { Sector } \\
\text { bancario }\end{array}$ & $\begin{array}{l}\text { Estructura de } \\
\text { Mercado }\end{array}$ & Índice de Lerner & Estru. Lerner & $\begin{array}{l}\text { Datos de } \\
\text { Estructura } \\
\text { Financiera }\end{array}$ & $\begin{array}{c}\text { Tasa de } \\
\text { crecimiento } \\
(\%)\end{array}$ \\
\hline
\end{tabular}

Fuente: Elaboración propia.

El segundo conjunto se centra en los mercados financieros en este grupo se consideran las dimensiones: acceso, profundidad, eficiencia, estabilidad y rentabilidad. Para la dimensión profundidad se utiliza la variable, capitalización del mercado de valores con respecto al PIB 
(P. market_cap) valores más altos para esta variable indican un mayor nivel de profundidad de los mercados financieros. La dimensión acceso se evalúa mediante el número de empresas listadas por millón de personas (A. list_comp), mayor número de empresas listadas señalan un mayor acceso a los mercados financieros. La dimensión eficiencia esta medida por la variable índice de rotación del mercado accionario(E.market_turn) niveles más altos para esta variable denotan mayor eficiencia del mercado accionario. La dimensión rentabilidad considera el rendimiento año a año del mercado accionario(R.market_ret), valores más altos para esta variable significan mayor obtención de ganancias. Finalmente, la dimensión estabilidad se evalúa mediante la variable volatilidad del precio de las acciones (Est.price_vol), altos niveles en esta variable apuntan a que existe una mayor variación de los precios de las acciones.

Tabla 3. Variables de los mercados financieros.

\begin{tabular}{|c|c|c|c|c|c|}
\hline Tipo & Dimensión & Variable & Abreviatura & Fuente & $\begin{array}{l}\text { Unidad de } \\
\text { medida }\end{array}$ \\
\hline $\begin{array}{l}\text { Mercados } \\
\text { Financieros }\end{array}$ & Acceso & $\begin{array}{l}\text { Número de } \\
\text { compañías } \\
\text { listadas por } \\
\text { cada } 1,000,000 \\
\text { de personas }\end{array}$ & A.list_comp & $\begin{array}{l}\text { Base de } \\
\text { Datos de } \\
\text { Estructura } \\
\text { Financiera }\end{array}$ & $\begin{array}{c}\text { Tasa de } \\
\text { crecimiento } \\
(\%)\end{array}$ \\
\hline $\begin{array}{l}\text { Mercados } \\
\text { Financieros }\end{array}$ & Profundidad & $\begin{array}{l}\text { Capitalización } \\
\text { del mercado } \\
\text { accionario/PIB }\end{array}$ & P.market_cap & $\begin{array}{l}\text { Base de } \\
\text { Datos de } \\
\text { Estructura } \\
\text { Financiera }\end{array}$ & $\begin{array}{c}\text { Tasa de } \\
\text { crecimiento } \\
(\%)\end{array}$ \\
\hline $\begin{array}{l}\text { Mercados } \\
\text { Financieros }\end{array}$ & Eficiencia & $\begin{array}{l}\text { Rotación del } \\
\text { mercado } \\
\text { accionario }\end{array}$ & E.market_turn & $\begin{array}{l}\text { Base de } \\
\text { Datos de } \\
\text { Estructura } \\
\text { Financiera }\end{array}$ & $\begin{array}{c}\text { Tasa de } \\
\text { crecimiento } \\
(\%)\end{array}$ \\
\hline $\begin{array}{l}\text { Mercados } \\
\text { Financieros }\end{array}$ & Rentabilidad & $\begin{array}{l}\text { Rendimiento del } \\
\text { mercado } \\
\text { accionario }\end{array}$ & R.market_ret & $\begin{array}{l}\text { Base de } \\
\text { Datos de } \\
\text { Estructura } \\
\text { Financiera }\end{array}$ & $\begin{array}{c}\text { Tasa de } \\
\text { crecimiento } \\
(\%)\end{array}$ \\
\hline $\begin{array}{l}\text { Mercados } \\
\text { financieros }\end{array}$ & Estabilidad & $\begin{array}{l}\text { Volatilidad del } \\
\text { precio de las } \\
\text { acciones }\end{array}$ & Est.price_vol & $\begin{array}{l}\text { Base de } \\
\text { Datos de } \\
\text { Estructura } \\
\text { Financiera }\end{array}$ & $\begin{array}{c}\text { Tasa de } \\
\text { crecimiento } \\
(\%)\end{array}$ \\
\hline
\end{tabular}

Fuente: Elaboración propia.

Para controlar otros factores que podrían impactar la desigualdad del ingreso en ambos conjuntos de variables se utilizan: gasto del gobierno (Cont.gov), apertura comercial (Cont.trade) e inflación (Cont.Inf). El gasto de gobierno se incorpora para examinar el efecto de 
las políticas públicas sobre la desigualdad del ingreso así mismo se incluye a la variable apertura comercial para estimar el efecto de la globalización sobre la desigualdad del ingreso. El signo esperado para las dos variables es negativo. Por último, la variable inflación mide la estabilidad de la economía y se espera que el signo sea positivo. Se pueden observar las variables y sus abreviaturas en las Tabla 1, 2, 3 y 4.

Tabla 4. Variables de control

\begin{tabular}{|l|l|l|l|l|c|}
\hline Tipo & Dimensión & Variable & Abreviatura & Fuente & $\begin{array}{l}\text { Unidad de } \\
\text { medida }\end{array}$ \\
\hline Control & $\begin{array}{l}\text { Macro- } \\
\text { económica }\end{array}$ & Inflación & Cont.Inf & $\begin{array}{l}\text { Datos de } \\
\text { Estructura } \\
\text { Financiera }\end{array}$ & $\begin{array}{c}\text { Tasa de } \\
\text { crecimiento } \\
(\%)\end{array}$ \\
\hline Control & $\begin{array}{l}\text { Macro- } \\
\text { económica }\end{array}$ & $\begin{array}{l}\text { Gasto de } \\
\text { gobierno/PIB }\end{array}$ & Cont.gov & $\begin{array}{l}\text { Indicadores } \\
\text { de } \\
\text { Desarrollo } \\
\text { Mundial }\end{array}$ & $\begin{array}{l}\text { Tasa de } \\
\text { crecimiento } \\
(\%)\end{array}$ \\
\hline Control & $\begin{array}{l}\text { Macro- } \\
\text { económica }\end{array}$ & $\begin{array}{l}\text { Apertura } \\
\text { comercial/PIB }\end{array}$ & Cont.trade & $\begin{array}{l}\text { Indicadores } \\
\text { de } \\
\text { Desarrollo } \\
\text { Mundial }\end{array}$ & $\begin{array}{c}\text { Tasa de } \\
\text { crecimiento } \\
(\%)\end{array}$ \\
\hline
\end{tabular}

Fuente: Elaboración propia.

Cabe destacar que todas las variables dependientes, independientes y de control están expresadas en tasa de crecimiento.

En las tablas 5 y 6 se muestran las estadísticas descriptivas de los dos conjuntos de variables (sector bancario y mercados financieros).

Tabla 5. Estadísticas descriptivas del sector bancario.

\begin{tabular}{|c|c|c|c|c|}
\hline Variable & $\begin{array}{c}\text { Media } \\
\mathbf{( \% )}\end{array}$ & $\begin{array}{c}\text { Desviación } \\
\text { Estándar (\%) }\end{array}$ & $\begin{array}{c}\text { Máximo } \\
\mathbf{( \% )}\end{array}$ & $\begin{array}{c}\text { Mínimo } \\
\text { (\%) }\end{array}$ \\
\hline Variables dependientes & \multicolumn{5}{|l|}{} \\
\hline Des.Atkinson & .7 & 11.6 & 183.4 & -62.7 \\
\hline Des.Gini & .3 & 6.5 & 81.7 & -42.9 \\
\hline Des.m2m & .07 & 4.8 & 64.5 & -46.7 \\
\hline Des.Palma & .9 & 16 & 279.1 & -96.2 \\
\hline Des.Theil & 1 & 15.7 & 270.6 & -68.9 \\
\hline Variables independientes & \multicolumn{5}{|l|}{} \\
\hline P.Credit & 905.5 & 32326 & 1187431.9 & -99.9 \\
\hline E.net_interest & 4.9 & 70.8 & 1903.1 & -90.3 \\
\hline E.return & -7.7 & 1047.2 & 17401.2 & -28054.7 \\
\hline Estab.Z_score & 21.2 & 463.4 & 14586.7 & -1487.3 \\
\hline Estab.cred_dep & 1.3 & 11.5 & 169.5 & -40.9 \\
\hline Estru.Conc & .03 & 10.6 & 76.1 & -61.5 \\
\hline
\end{tabular}




\begin{tabular}{|c|c|c|c|c|}
\hline Estru. Lerner & 21.2 & 831.6 & 29055.2 & -7097.8 \\
\hline Variables de control \\
\hline Cont. Inf & 5.9 & 12.4 & 251.8 & -15.8 \\
\hline Cont.gov & .6 & 8.2 & 88.6 & -51.9 \\
\hline Cont. trade & 1.4 & 9.9 & 149 & -41.5 \\
\hline
\end{tabular}

Fuente: Elaboración propia.

Tabla 6. Estadísticas descriptivas de los mercados financieros.

\begin{tabular}{|c|l|l|l|l|}
\hline Variable & \multicolumn{1}{|c|}{$\begin{array}{c}\text { Media } \\
\text { (\%) }\end{array}$} & $\begin{array}{c}\text { Desviación } \\
\text { Estándar (\%) }\end{array}$ & \multicolumn{1}{|c|}{$\begin{array}{c}\text { Máximo } \\
\text { (\%) }\end{array}$} & \multicolumn{1}{c|}{$\begin{array}{c}\text { Mínimo } \\
\text { (\%) }\end{array}$} \\
\hline Variables dependientes & .7 & 11.2 & 147.9 & -62.7 \\
\hline Des. Atkinson & .2 & 6.2 & 75.1 & -42.9 \\
\hline Des.Gini & .2 & 4.5 & 64.5 & -46.7 \\
\hline Des.m2m & .1 & 15.4 & 279.1 & -84.7 \\
\hline Des.Palma & 1 & 14.7 & 221.9 & -68.9 \\
\hline Des.Theil & .9 & \multicolumn{5}{|l|}{} \\
\hline Variables independientes & 7.7 & 1691.8 & -172.3 \\
\hline A.list_comp & 4.7 & 3695.6 & -283.44 \\
\hline P.market_cap & 10.78 & 124.1 & 10147.3 & -234.3 \\
\hline E.market_turn & 25.3 & 358.1 & 24929.9 & -26732.1 \\
\hline R.market_ret & -58.1 & 1277.3 & 565.7 & -164 \\
\hline Est.price_vol & 3 & 38 & 115.3 & -15.8 \\
\hline Variables de control & 5 & 88.6 & -51.9 \\
\hline Cont. Inf & 5 & 8.6 & 149 & -41.5 \\
\hline Cont.gov & .4 & 6.6 & \\
\hline Cont.trade & 1.4 & 9.9 &
\end{tabular}

Fuente: Elaboración propia.

\section{Metodología}

Para examinar la relación entre las diferentes dimensiones del sector bancario y de las características de los mercados financieros con respecto a la desigualdad del ingreso se especifican los siguientes modelos:

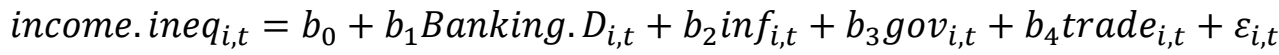

$$
\begin{aligned}
& \text { income. } \text { ineq }_{i t}=b_{0}+b_{1} \text { Fin. Markets }+b_{2} \text { inf }+b_{3} \text { gov }+b_{4} \text { trade }+\varepsilon_{i t}
\end{aligned}
$$

De manera específica se estiman diez modelos, cinco modelos para el sector bancario y cinco para los mercados financieros. El modelo empleado como base para analizar la relación entre desigualdad del ingreso y las diferentes dimensiones de los mercados financieros es el siguiente:

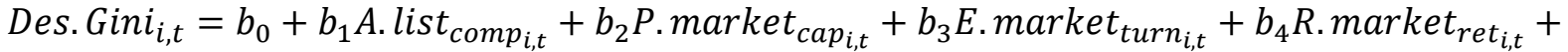

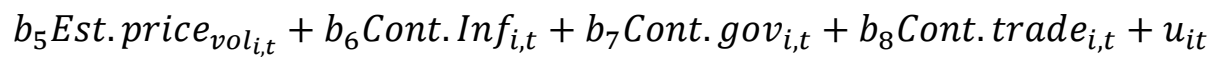


Dicho modelo se estima cuatro veces más utilizando un índice de desigualdad del ingreso diferente cada vez. Donde $i$ representa el país y $t$ el año. Los índices de desigualdad que se emplean son el índice de Gini, el índice de Atkinson, el índice de Theil, la proporción Palma y la proporción Media a Mediana.

Por otro lado, para analizar la relación entre la desigualdad del ingreso y el sector bancario se utiliza la siguiente ecuación:

$$
\begin{aligned}
& \text { Des.Gini } i_{i, t}=b_{0}+b_{1} \text { P.Credit } \text { Crt }_{i}+b_{2} \text { E.net_interest }_{i, t}+b_{3} \text { E.return }_{i, t}+b_{4}{\text { Estab. } Z_{-} \text {score }_{i, t}+}+
\end{aligned}
$$

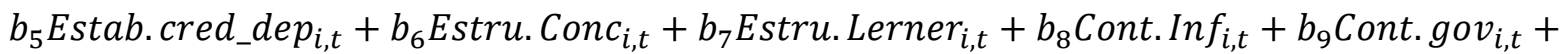

$$
\begin{aligned}
& b_{10} \text { Cont. trade } e_{i, t}+u_{i t}
\end{aligned}
$$

Al igual que con el modelo anterior este modelo se estima en cinco ocasiones y en cada ocasión se emplea uno de los índices de desigualdad ya mencionados como variables dependientes. El método de estimación que se utiliza es el de Mínimos Cuadrados Ordinarios. Por otro lado, como prueba de robustez se utiliza el método de Mínimo Cuadrados Generalizados Estimados (EGLS) con efectos aleatorios de dos vías. Este método es eficiente cuando se estiman los parámetros de observaciones que muestran heterocedasticidad y cuando además existe cierto grado de correlación entre las variables. El método considera que en algunos casos no se conoce la matriz de covarianza $(\Sigma)$. Por lo tanto, esta matriz debe ser estimada. Para obtener dicha matriz se considera el siguiente modelo:

$$
y_{t}=\beta X+u_{t}
$$

Donde $y_{t}$ es la variable dependiente, $\beta$ es el coeficiente a estimar, $X$ es una matriz $n * p$ de variables independientes y $u_{t}$ es el término de error. Con $t=1, \ldots . N$. Al tomar la media de todas las observaciones de la variable dependiente y el término de error. El modelo se transforma en la siguiente expresión:

$$
\bar{y}=\beta X+\bar{u}
$$

Donde $\bar{y}$ y $u$ son los promedios de la variable dependiente y el término de error respectivamente. Por lo tanto, el estimador imparcial de $\Sigma$ estará dado por la siguiente ecuación:

$$
\hat{\Sigma}=(N-1)^{-1} \sum_{t=1}^{N}\left(y_{t}-\bar{y}\right)\left(y_{t}-\bar{y}\right)^{T}
$$

Se conoce que $\widehat{\Sigma}$ es un estimado de $\Sigma$ entonces el estimado de mínimos cuadrados generalizados para $\beta$ esta dado por la siguiente ecuación (Sen y Srivastava, 1990):

$$
\beta_{E G L S}=\left(X^{T} \hat{\Sigma}^{-1} X\right)^{-1} X^{T} \hat{\Sigma}^{-1} \bar{y}
$$

En este método se asume que el término de error $u_{t}$ es un término de perturbación puro. Sin embargo, cuando se considera un método de dos vías el término de error está compuesto por: efectos 
individuales no observables, efectos no observables en el tiempo y un término de perturbación pura. Por tanto, el término de error se puede expresar de la siguiente manera:

$$
u_{i t}=\theta_{i}+\tau_{t}+\varepsilon_{i t}
$$

Donde $\theta_{i}$ y $\tau_{t}$ son los efectos individuales no observables y efectos no observables en el tiempo respectivamente. Si $\theta_{i}$ o $\tau_{t}$ es diferente de cero entonces se trata de un modelo de una vía para el componente de error. Si ambos $\theta_{i}$ y $\tau_{t}$ son diferentes de cero se considera un modelo de dos vías para el componente de error. Existe dos métodos para estimar el término de error de la ecuación (9) efectos fijos y efectos aleatorios. La base de datos que se considera para este análisis está conformada por información a nivel país. Y cada país tiene características específicas propias que no se pueden observar directamente los cuales podrían influir sobre la desigualdad del ingreso. Estas características pueden ser de tipo: cultural y religioso entre otros. Asimismo, existen ciertos factores que ocurren en el tiempo como huelgas, desastres naturales que tampoco se pueden observar directamente. Po otro lado, los resultados de la prueba conjunta de efectos aleatorios correlacionados presentan evidencia en favor del uso de efectos aleatorios de dos vías como se puede observar en las tablas 8 y 10. Por tanto, se considera un modelo de efectos aleatorios de dos vías el cual considera la inclusión de dos variables dummies una para las características específicas de cada país y otra para los efectos del tiempo (Kaizoji y Miyano, 2016). El modelo se expresa de la siguiente forma:

$$
y_{i, t}=\theta_{i}+\tau_{t}+\beta_{1} x_{i, t}+\beta_{2} C_{i, t}+\varepsilon_{i t}
$$

Donde $\theta_{i}$ y $\tau_{t}$ son los características específicas de cada país y los efectos temporales respectivamente, $y_{i, t}$ incorpora las variables de desigualdad, $x_{i, t}$ incluye las variables relacionados al sistema financiero (variables del mercado financiero y del sector bancario) y $C_{i, t}$ son las variables de control. Por tanto, los modelos a estimar son los siguientes:

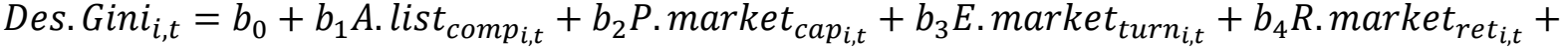

$$
\begin{aligned}
& b_{5} \text { Est.price }_{\text {vol }_{i, t}}+b_{6} \text { Cont. Inf }_{i, t}+b_{7} \text { Cont. gov }_{i, t}+b_{8} \text { Cont.trade }_{i, t}+\theta_{i}+\tau_{t}+\varepsilon_{i t}
\end{aligned}
$$

para los mercados financieros $\mathrm{y}$

$$
\begin{aligned}
& \text { Des.Gini }{ }_{i, t}=b_{0}+b_{1} \text { P.Credit } \text { Cr,t }_{i}+b_{2} \text { E. net }_{\text {interest }_{i, t}}+b_{3} \text { E.return }_{i, t}+b_{4} \text { Estab. }_{\text {score }_{i, t}}+
\end{aligned}
$$

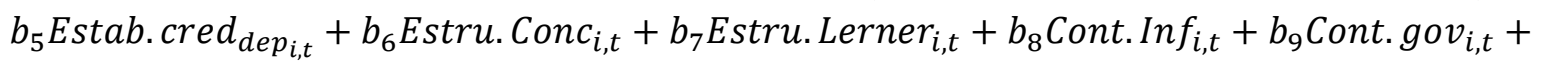

$$
\begin{aligned}
& b_{10} \text { Cont.trade } e_{i, t}+\theta_{i}+\tau_{t}+\varepsilon_{i t}
\end{aligned}
$$

para el sector bancario. Ambos modelos se estiman en cinco ocasiones diferentes una vez por cada índice de desigualdad.

\section{Resultados}

Se estimaron cuatro conjuntos de modelos, estos conjuntos se dividen en dos: en el primer conjunto se analiza la relación entre las diferentes dimensiones del sector bancario y la desigualdad del 
ingreso, en el segundo conjunto se analiza la relación entre las diferentes características de los mercados financieros y la desigualdad del ingreso. En ambos conjuntos se utilizan los métodos Mínimos Cuadrados Ordinarios y Mínimos Cuadrados Generalizados Estimados con efectos aleatorios de dos vías como prueba de robustez.

Tabla 7. Resultados para la muestra del sector bancario empleando el método de Mínimos Cuadrados Ordinarios

\begin{tabular}{|c|c|c|c|c|c|}
\hline & Variables deper & lientes & & & \\
\hline $\begin{array}{l}\text { Observaciones: } \\
1050\end{array}$ & $\begin{array}{c}\text { Des. Atkinson } \\
{[1]}\end{array}$ & $\begin{array}{c}\text { Des.Gini } \\
\text { [2] }\end{array}$ & $\begin{array}{c}\text { Des. } m 2 m \\
\text { [3] }\end{array}$ & $\begin{array}{c}\text { Des. Palma } \\
\text { [4] }\end{array}$ & $\begin{array}{c}\text { Des.Theil } \\
\text { [5] }\end{array}$ \\
\hline \multicolumn{6}{|l|}{ Variables } \\
\hline \multicolumn{6}{|c|}{ Variables independientes } \\
\hline P.Credit & $0.0001^{* * *}$ & $0.00006^{* * *}$ & $0.00003^{* * *}$ & $0.0001^{* * *}$ & $0.0002^{* * *}$ \\
\hline E.net_interest & 0.002 & 0.003 & 0.0004 & 0.002 & 0.004 \\
\hline E.return & $0.0005^{*}$ & $0.0002^{*}$ & $0.0002^{*}$ & $0.0007^{*}$ & $0.0007^{* *}$ \\
\hline Estab.Z_score & 0.0001 & 0.0002 & 0.00009 & 0.0003 & 0.0004 \\
\hline Estab.cred_dep & $-0.05^{* *}$ & -0.02 & -0.004 & -0.05 & $-0.05^{*}$ \\
\hline Estru.Conc & 0.002 & 0.01 & 0.01 & 0.01 & 0.01 \\
\hline Estru.Lerner & $0.0005^{*}$ & 0.0002 & 0.0001 & 0.0005 & 0.0005 \\
\hline \multicolumn{6}{|l|}{ Variables de control } \\
\hline Cont. Inf & 0.02 & 0.005 & -0.001 & 0.03 & 0.04 \\
\hline Cont.gov & 0.001 & -0.004 & -0.005 & 0.01 & -0.01 \\
\hline Cont. trade & -0.04 & -0.02 & -0.004 & -0.004 & -0.05 \\
\hline \multicolumn{6}{|l|}{ Estadísticas } \\
\hline$R^{2}$ & 0.26 & 0.16 & 0.05 & 0.14 & 0.31 \\
\hline Durbin - Watson & 2.21 & 2.30 & 2.15 & 2.009 & 2.24 \\
\hline
\end{tabular}

Fuente: Elaboración propia.

En la tabla 7 se muestran los resultados de las estimaciones que analizan la relación entre las características del sector bancario y la desigualdad del ingreso empleando el método de Mínimos Cuadrados Ordinarios.

Los resultados para las variables independientes muestran que para la dimensión profundidad la cual esta evaluada por P.Credit es estadísticamente significativa al 1\% en todos los modelos. Un aumento de $1 \%$ en esta variable significa un aumento de la desigualdad del .01\%, .006\%, $.003 \%, .01 \%$ y .02\% en la desigualdad del ingreso para los modelos [1], [2], [3], [4] y [5]. La dimensión eficiencia esta evaluada por E.net_interest y E.return se encuentra que la primera variable no es estadísticamente significativa. Mientras que la variable E.return es estadísticamente significativa al $5 \%$ en el modelo [5] y al $10 \%$ en los modelos [1], [2], [3] y [4]. Un aumento de $1 \%$ en esta variable significa un aumento de $.05 \%, .02 \%, .02 \%, .07 \%$ y $.07 \%$ en la desigualdad del ingreso para los modelos [1], [2], [3], [4] y [5] respectivamente. La dimensión estabilidad considera dos variables Estab.Z_score y Estab.cred_dep. Por un lado, la variable Estab.Z_score no es estadísticamente 
significativa y por otro lado, Estab.cred_dep es estadísticamente significativa al 5\% en el modelo [1] y al 10\% en el modelo [5]. Un aumento del 1\% para Estab.cred_dep significa una reducción del $5 \%$ en la desigualdad del ingreso en el modelo [1] y una reducción del 5\% para el modelo [5]; en los modelos [2] [3] y [4] la variable no es estadísticamente significativa. La dimensión estructura del mercado está compuesta por Estru. Conc y Estru.Lerner de estas variables solo Estru. Lerner es estadísticamente significativa al 10\% en el modelo [1]. Un aumento de 1\% en Estru. Lerner conlleva un aumento de .05\% de la desigualdad del ingreso.

Los resultados de las variables de control muestran que Cont. Inf, Cont.gov y Cont.trade no son estadísticamente significativas.

Tabla 8. Resultados para la muestra del sector bancario empleando el método de Mínimos Cuadrados Generalizados Estimados con efectos aleatorios de dos vías.

\begin{tabular}{|c|c|c|c|c|c|}
\hline \multirow[b]{2}{*}{$\begin{array}{l}\text { Observaciones: } \\
1050\end{array}$} & \multicolumn{5}{|c|}{ Variables dependientes } \\
\hline & $\begin{array}{c}\text { Des. Atkinson } \\
\text { [6] }\end{array}$ & $\begin{array}{c}\text { Des.Gini } \\
\text { [7] }\end{array}$ & $\begin{array}{c}\text { Des. } m 2 m \\
{[8]}\end{array}$ & $\begin{array}{c}\text { Des. Palma } \\
\text { [9] }\end{array}$ & $\begin{array}{c}\text { Des.Theil } \\
{[10]}\end{array}$ \\
\hline \multicolumn{6}{|l|}{ Variables } \\
\hline intercepto & 0.001 & -0.0005 & $-0.003^{*}$ & -0.002 & -0.0002 \\
\hline \multicolumn{6}{|c|}{ Variables independientes } \\
\hline P.Credit & $0.0001^{* * *}$ & $0.00006^{* * *}$ & $0.00003^{* * *}$ & $0.0001^{* * *}$ & $0.0002^{* * *}$ \\
\hline E.net_interest & 0.002 & 0.003 & 0.0008 & 0.002 & 0.004 \\
\hline E.return & $0.0005^{* *}$ & $0.0002^{*}$ & $0.0002^{*}$ & $0.0007^{*}$ & $0.0007^{* *}$ \\
\hline Estab.Z_score & 0.0001 & 0.0002 & 0.0001 & 0.0003 & 0.0004 \\
\hline Estab.cred_dep & $-0.05^{* *}$ & -0.02 & -0.001 & -0.05 & -0.05 \\
\hline Estru.Conc & 0.002 & 0.01 & 0.01 & 0.01 & 0.01 \\
\hline Estru.Lerner & $0.0005^{*}$ & 0.0002 & 0.0001 & 0.0005 & 0.0005 \\
\hline \multicolumn{6}{|l|}{ Variables de control } \\
\hline Cont.Inf & 0.01 & 0.006 & 0.008 & 0.04 & 0.04 \\
\hline Cont.gov & -0.00006 & -0.004 & -0.001 & 0.02 & -0.01 \\
\hline Cont.trade & $-0.04^{*}$ & -0.02 & -0.0006 & -0.001 & -0.05 \\
\hline \multicolumn{6}{|l|}{ Estadísticas } \\
\hline$R^{2}$ & 0.26 & 0.16 & 0.06 & 0.14 & 0.31 \\
\hline Durbin-Watson & 2.21 & 2.3 & 2.16 & 2.01 & 2.24 \\
\hline $\begin{array}{c}\text { Prueba conjunta } \\
\text { de efectos } \\
\text { aleatorios } \\
\text { correlacionados }\end{array}$ & .71 & .95 & .99 & .97 & .82 \\
\hline
\end{tabular}

***,**,* Estadísticamente significativo al 1\%,5\% y $10 \%$ respectivamente.

Fuente: Elaboración propia.

Así mismo, en la tabla 8 se muestran los resultados de las estimaciones que analizan la relación entre las características del sector bancario y la desigualdad del ingreso empleando el método de Mínimos Cuadrados Generalizados Estimados con efectos aleatorios de dos vías.

Los resultados para las variables independientes muestran que para la dimensión profundidad la cual esta evaluada por P.Credit es estadísticamente significativa al $1 \%$ en todos los 
modelos. Un aumento de $1 \%$ en esta variable significa un aumento de la desigualdad del .01\%, .006\%, $.003 \%, .01 \%$ y $.02 \%$ en la desigualdad del ingreso para los modelos [6], [7], [8], [9] у [10]. La dimensión eficiencia esta evaluada por E. net_interest y E.return; los resultados muestran que la primera variable no es estadísticamente significativa. Mientras que la variable E.return es estadísticamente significativa al 5\% en los modelos [6] y [10] y al 10\% en los modelos [7], [8] y [9]. Un aumento de $1 \%$ para esta variable significa un aumento de .05\%, .02\%, .02\%, .07\% y .07\% en la desigualdad del ingreso en los modelos [6], [7], [8], [9] y [10]. La dimensión estabilidad considera dos variables Estab.Z_score y Estab.cred_dep. La variable Estab.Z_score no es estadísticamente significativa. Por otro lado, Estab.cred_dep es estadísticamente significativa al 5\% en el modelo [6]. Un aumento del 1\% para Estab.cred_dep significa una reducción de la desigualdad del ingreso del 5\% para el modelo [6]. La dimensión estructura del mercado está compuesta por Estru.Conc y Estru.Lerner de estas variables solo Estru.Lerner es estadísticamente significativa al $10 \%$ en el modelo [6]. Un aumento de 1\% en Estru. Lerner conlleva un aumento de .05\% de la desigualdad del ingreso.

Los resultados de las variables de control muestran que Cont.Inf y Cont.gov no son estadísticamente significativas. Mientras que la variable Cont.trade tiene el signo esperado y es estadísticamente significativa al $10 \%$ en el modelo [6].

Los resultados de ambas estimaciones coinciden en lo general. Encontrándose que para ambas estimaciones y para todas las medidas de desigualdad del ingreso la variable P. Credit aporta evidencia a favor de la hipótesis de aumento de la desigualdad. Así mismo, la variable E.return la cual analiza la dimensión eficiencia, aporta evidencia a favor de la hipótesis del aumento de la desigualdad cuando consideramos ambos métodos de estimación. Por otro lado, los resultados empleando los dos modelos coinciden en que la variable Estab.cred_dep aporta evidencia a favor de la hipótesis de la reducción de la desigualdad. Mientras que para la variable Estru. Lerner se observa un aumento de la desigualdad del ingreso cuando disminuye el nivel de competencia en el sector bancario.

Tabla 9. Resultados de la muestra mercados financieros empleando el método de Mínimos Cuadrados Ordinarios

\begin{tabular}{|c|c|c|c|c|c|}
\hline \multicolumn{6}{|c|}{ Método: Mínimos Cuadrados Ordinarios } \\
\hline & \multicolumn{5}{|c|}{ Variables dependientes } \\
\hline $\begin{array}{l}\text { Observaciones: } \\
798\end{array}$ & $\begin{array}{c}\text { Des. Atkinson } \\
{[11]}\end{array}$ & $\begin{array}{c}\text { Des.Gini } \\
\text { [12] }\end{array}$ & $\begin{array}{c}\text { Des. } m 2 m \\
{[13]}\end{array}$ & $\begin{array}{c}\text { Des. Palma } \\
{[14]}\end{array}$ & $\begin{array}{c}\text { Des.Theil } \\
\text { [15] }\end{array}$ \\
\hline \multicolumn{6}{|l|}{ Variables } \\
\hline \multicolumn{6}{|c|}{ Variables independientes } \\
\hline A. list_comp & $0.02^{* * *}$ & $0.01^{* * *}$ & $0.007^{* * *}$ & $0.03^{* * *}$ & $0.03^{* * *}$ \\
\hline P.market_cap & 0.0003 & -0.001 & -0.0009 & -0.002 & -0.005 \\
\hline E.market_turn & -0.0009 & -0.0005 & -0.0004 & -0.001 & -0.001 \\
\hline R.market_ret & 0.00008 & 0.00005 & 0.00001 & 0.0001 & 0.0001 \\
\hline Est.price_vol & -0.007 & -0.006 & -0.002 & -0.01 & -0.01 \\
\hline \multicolumn{6}{|c|}{ Variables de control } \\
\hline Cont.Inf & $0.06^{*}$ & 0.03 & 0.01 & $0.1^{*}$ & $0.12^{* *}$ \\
\hline Cont.gov & 0.04 & 0.002 & 0.01 & 0.1 & 0.03 \\
\hline
\end{tabular}




\begin{tabular}{|c|c|c|c|c|c|}
\hline Cont.trade & -0.07 & $-0.04^{* *}$ & -0.01 & -0.009 & -0.07 \\
\hline \multicolumn{6}{|l|}{ Estadísticas } \\
\hline$R^{2}$ & 0.04 & 0.03 & 0.01 & 0.03 & 0.05 \\
\hline Durbin - Watson & 2.22 & 2.19 & 2.20 & 1.98 & 2.24 \\
\hline
\end{tabular}

Los resultados de la estimación empleando las características de los mercados financieros con el método de estimación Mínimos Cuadrados Ordinarios se muestran en la tabla 9. Los resultados para las variables independientes se muestran a continuación. La variable A.list_comp que corresponde a la dimensión acceso, es estadísticamente significativa al $1 \%$ en todo los modelos. Un aumento de $1 \%$ en esta variable significa un aumento del $2 \%, 1 \%, 7 \%$, $3 \%$ y $3 \%$ en la desigualdad del ingreso en los modelos [11], [12], [13], [14] y [15] respectivamente. El resto de las dimensiones consideradas: profundidad, eficiencia, rentabilidad y estabilidad no son estadísticamente significativas. En cuanto a las variables de control Cont. Inf es estadísticamente significativa al 5\% en el modelo [15] y al 10\% en los modelos [11] y [14]. Cont. Inf muestra el signo esperado. Por otro lado, Cont. gov y Cont. trade no son estadísticamente significativas.

Tabla 10. Resultados de la muestra mercados financieros empleando el método de Mínimos Cuadrados Generalizados Estimados con efectos aleatorios de dos vías.

\begin{tabular}{|c|c|c|c|c|c|}
\hline \multicolumn{6}{|c|}{ Método: Mínimos Cuadrados Generalizados Estimados con efectos aleatorios de dos vías } \\
\hline \multirow[b]{2}{*}{ Observaciones: 798} & \multicolumn{5}{|c|}{ Variables dependientes } \\
\hline & $\begin{array}{c}\text { Des. Atkinson } \\
{[16]}\end{array}$ & $\begin{array}{c}\text { Des.Gini } \\
\text { [17] }\end{array}$ & $\begin{array}{c}\text { Des. } m 2 m \\
{[18]}\end{array}$ & $\begin{array}{c}\text { Des.Palma } \\
\text { [19] }\end{array}$ & $\begin{array}{c}\text { Des. Theil } \\
\text { [20] }\end{array}$ \\
\hline \multicolumn{6}{|l|}{ Variables } \\
\hline intercepto & -0.002 & -0.002 & $-0.003^{*}$ & -0.005 & -0.006 \\
\hline \multicolumn{6}{|c|}{ Variables independientes } \\
\hline A. list_comp & $0.02^{* * *}$ & $0.01^{* * *}$ & $0.007^{* * *}$ & $0.03^{* * *}$ & $0.03^{* * *}$ \\
\hline P.market_cap & 0.005 & 0.001 & 0.001 & 0.003 & 0.007 \\
\hline E.market_turn & -0.0008 & 0.0005 & -0.0004 & -0.001 & -0.001 \\
\hline R.market_ret & 0.00009 & 0.00005 & -0.000002 & 0.0001 & 0.0001 \\
\hline Est.price_vol & -0.006 & -0.005 & -0.002 & -0.01 & -0.008 \\
\hline \multicolumn{6}{|l|}{ Variables de control } \\
\hline Cont.Inf & $0.08^{*}$ & $0.05^{* *}$ & 0.02 & $0.13^{* *}$ & $0.15^{* * *}$ \\
\hline Cont.gov & 0.04 & 0.007 & 0.01 & 0.1 & 0.04 \\
\hline Cont.trade & -0.05 & $-0.03^{*}$ & -0.01 & -0.003 & -0.07 \\
\hline \multicolumn{6}{|l|}{ Estadísticas } \\
\hline$R^{2}$ & 0.04 & 0.03 & 0.02 & 0.03 & 0.05 \\
\hline Durbin - Watson & 2.22 & 2.19 & 2.21 & 1.98 & 2.23 \\
\hline $\begin{array}{c}\text { Prueba conjunta } \\
\text { de efectos } \\
\text { aleatorios } \\
\text { correlacionados }\end{array}$ & 0.99 & 0.94 & 0.96 & 0.99 & 0.98 \\
\hline
\end{tabular}

***,**,* Estadísticamente significativo al 1\%,5\% y 10\% respectivamente.

Fuente: Elaboración propia 
Los resultados de la estimación empleando variables de los mercados financieros con el método de estimación Mínimos Cuadrados Generalizados Estimados con efectos aleatorios de dos vías se muestran en la tabla 10. Los resultados para las variables independientes se muestran a continuación. La variable $A$. list_comp que corresponde a la dimensión acceso, es estadísticamente significativa al $1 \%$ en los cinco modelos. Un aumento de $1 \%$ en esta variable significa un aumento del $2 \%, 1 \%, .7 \%, 3 \%$ y $3 \%$ en la desigualdad del ingreso en los modelos [16], [17], [18], [19] у [20] respectivamente. Las dimensiones: profundidad, eficiencia, estabilidad y rentabilidad no son estadísticamente significativas. En cuanto a las variables de control Cont. Inf cuenta con el signo esperado y es estadísticamente significativa al 1\% en el modelo [20], al 5\% en los modelos [17] y [19] y al $10 \%$ en el modelo [16]. Mientras que Cont.gov no es estadísticamente significativa. Por otro lado, la variable Cont.trade es estadísticamente significativa al 10\% en el modelo [17], la variable exhibe el signo esperado.

Por tanto, los resultados para la variable A.list_comp aportan evidencia que apoya la hipótesis de un aumento de la desigualdad del ingreso. Por otro lado, para el resto de las dimensiones no se encontró evidencia significativa que apoye a alguna de las hipótesis en estudio. Estos resultados concuerdan con los obtenidos con el método de Mínimos Cuadrados Ordinarios. Cabe mencionar que en los resultados de las estimaciones de la muestra mercado financieros se obtuvieron coeficientes de determinación bajos. Por tanto, se considera que el sector bancario tiene un mayor impacto sobre la desigualdad del ingreso debido a que exhibe coeficientes de determinación más altos.

En resumen, se encuentra que la dimensión profundidad tiene una relación positiva con la desigualdad del ingreso. La dimensión profundidad del sector bancario evaluada por la variable P. Credit muestra evidencia de una relación positiva con las medidas de desigualdad del ingreso. Para la dimensión eficiencia se encontró evidencia de una relación positiva entre la variable del sector bancario E.return y la desigualdad del ingreso. En el caso de la dimensión acceso para los mercados financieros representada por la variable A. list_comp presenta evidencia a favor de una relación positiva con respecto de la desigualdad del ingreso. En cuanto a la dimensión estabilidad del sector bancario existe evidencia de una relación negativa entre Estab.cred_dep y la desigualdad del ingreso. Por último, los resultados de la variable Estru. Lerner muestran evidencia de que existe un aumento de la desigualdad del ingreso cuando disminuye el nivel de competencia en el sector bancario. Cabe destacar que en solo un modelo de los cinco que se estimaron; las variables Estab.cred_dep y Estru. Lerner son estadísticamente significativas cuando se emplea el método de Mínimos Cuadrados Generalizados Estimados con efectos aleatorios de dos vía. Mientras que P.Credit, E.return, y A. list_comp son estadísticamente significativos en todos los modelos.

Por otro lado, los resultados antes mencionados tanto para el sector bancario como para los mercados financieros no coinciden con los obtenidos en el trabajo de Zhang y Naceur (2019) ellos examinan las dimensiones: profundidad, acceso, eficiencia, liberalización y estabilidad. Sus resultados muestran que todas las dimensiones con excepción de liberalización aportan evidencia a favor de la hipótesis de reducción de la desigualdad. De igual manera en el trabajo de Meniago y Asongu (2019) el cual se enfoca en países africanos se consideran las dimensiones: profundidad, eficiencia, estabilidad y actividad. Ellos encuentran que todas las dimensiones con la excepción de la dimensión estabilidad tienen un efecto reductor de la desigualdad. En contraste, los resultados de los trabajos Seven y Coskun (2016), de Haan y Sturm (2016) y Chiu y Lee (2019) concuerdan con los 
obtenidos en la presenta investigación. Cabe mencionar que los trabajos de Seven y Coskun (2016) y de Haan y Sturm (2016) consideran una sola dimensión del sistema financiero y no hace una distinción entre el sector bancario y los mercados financieros. Por otro lado, el trabajo de Chiu y Lee (2019) estudia la relación no lineal entre desarrollo del sistema financiero y desigualdad del ingreso, ellos encuentran evidencia de que el desarrollo financiero aumenta la desigualdad del ingreso para países de bajos ingresos.

Por últimos los resultados de la dimensión estructura de mercado del sector bancario concuerdan con el trabajo de Hsieh, Chen y Lin (2019) es decir encuentran evidencia de una relación positiva entre menor nivel de competencia en el sector bancario y la desigualdad del ingreso.

\section{Conclusiones}

Se analizó la relación entre desigualdad del ingreso y las diferentes dimensiones del sector bancario. De igual manera, se evalúa el nexo entre la desigualdad del ingreso y las características de los mercados financieros. Para lo cual, se emplearon los métodos econométricos: Mínimos Cuadrados Ordinarios y Mínimos Cuadrados Generalizados Estimados con efectos aleatorios de dos vías. Se estimaron cinco modelos diferentes uno por cada medida de desigualdad. El período que se analiza es del año 1999 al 2012. Para el análisis se consideran dos muestras: la primera está compuesta por 75 países para las dimensiones del sector bancario y la segunda muestra comprende 57 países para las características del mercado financiero. Los resultados para los mercados financieros muestran que la dimensión acceso aporta evidencia a favor de la hipótesis de un aumento de la desigualdad del ingreso. Por otro lado, los resultados para el sector bancario indican que para las dimensiones profundidad y eficiencia existe evidencia a favor de la hipótesis de un aumento de la desigualdad del ingreso. Por el contrario, la dimensión estabilidad presenta evidencia a favor de la hipótesis de la disminución de la desigualdad del ingreso. Así mismo, se encuentra evidencia de una relación positiva entre menores niveles de competencia y la desigualdad del ingreso. Esto significa que mayor competencia está relacionada con una menor desigualdad del ingreso. Por otro lado, se encuentra que el sector bancario tiene índices de correlación más altos que los encontrados para los mercados financieros.

En contraste con otras investigaciones como las de Meniago y Asongu (2019) y Zhang y Naceur (2019) donde se encuentra que todas las dimensiones del sistema financiero reducen la desigualdad del ingreso. De acuerdo a la evidencia encontrada en esta investigación, se debe reconsiderar el papel del sector bancario sobre todo en cuanto a la dimensión profundidad. Ya que la dimensión profundidad tiene el efecto de aumentar la desigualdad. Por tanto, se debería garantizar condiciones equitativas en la utilización de los servicios financieros, asimismo, mejorar el acceso y la calidad de la información acerca de los mismos. Por otro lado, alcanzar un alto nivel de educación financiera debe ser una prioridad para los países. Debido a que el sistema financiero es una espada de doble filo; el uso correcto de los servicios que este provee, permite a los individuos financiar sus proyectos, mientras que su mal uso puede derivar en problemas financieros. Por tanto, un mejor acceso a la información y un mayor nivel de educación financiera permitirá a los individuos aprovechar de manera eficiente los servicios financieros. Así mismo, esta situación puede estar asociado a un acceso desigual al crédito otorgado por parte del sector bancario. Una limitante de esta investigación es no incluir la dimensión acceso para el sector bancario, sin embargo, los datos no 
estaban disponibles para el período de tiempo considerado en la investigación. Por tanto, sería importante analizar el efecto del acceso al sector bancario sobre la desigualdad del ingreso. Por otro lado, también sería de interés examinar la relación entre desigualdad del ingreso y las diferentes dimensiones del sistema financiero utilizando diferentes subconjuntos de países.

\section{Referencias}

[1] Agnello, L., Mallick, S. K., y Sousa, R. M. (2012). Financial reforms and income inequality. Economics Letters, 116(3), 583-587. https://doi.org/10.1016/j.econlet.2012.06.005

[2] Altunbaş, Y. y Thornton, J. (2019). The impact of financial development on income inequality: A quantile regression approach. Economics Letters, 175, 51-56. https://doi.org/10.1016/j.econlet.2018.12.030

[3] Banerjee, A. V. y Newman, A. F. (1993). Occupational Choice and the Process of Development. Journal of Political Economy, 101(2), 274-298. https://doi.org/10.1086/261876

[4] Beck, T., de Jonghe, O. y Schepens, G. (2013). Bank Competition and Stability: Cross-Country Heterogeneity. SSRN Electronic Journal. https://doi.org/10.2139/ssrn.1890674

[5] Beck, T., Demirgüç-Kunt, A. y Levine, R. (2007). Finance, inequality and the poor. Journal of Economic Growth, 12(1), 27-49. https://doi.org/10.1007/s10887-007-9010-6

[6] Birdsall, N. y Meyer, C. J. (2015). The Median is the Message: A Good Enough Measure of Material Wellbeing and Shared Development Progress. Global Policy, 6(4), 343-357. https://doi.org/10.1111/1758-5899.12239

[7] Bjørnskov, C. (2015). Growth, Inequality and Economic Freedom: Evidence from the US States. SSRN Electronic Journal. https://doi.org/10.2139/ssrn.2684730

[8] Boix, C. (2008). Economic Roots of Civil Wars and Revolutions in the Contemporary World. World Politics, 60(3), 390-437. https://doi.org/10.1017/s0043887100009047

[9] Boyd, J. H. y de Nicoló, G. (2005). The Theory of Bank Risk Taking and Competition Revisited. The Journal of Finance, 60(3), 1329-1343. https://doi.org/10.1111/j.1540-6261.2005.00763.x

[10] Chiu, Y. B. y Lee, C. C. (2019) Financial development, income inequality, and country risk. Journal of International Money and Finance https://doi.org/10.1016/j.jimonfin.2019.01.001

[11] Clarke, G. R. G., Xu, L. C. y Zou, H. (2006). Finance and Income Inequality: What Do the Data Tell Us? Southern Economic Journal, 72(3), 578. https://doi.org/10.2307/20111834

[12] Cobham, A., Schlögl, L. y Sumner, A. (2016). Inequality and the Tails: the Palma Proposition and Ratio. Global Policy, 7(1), 25-36. https://doi.org/10.1111/1758-5899.12320

[13] de Haan, J. y Sturm, J.-E. (2016). Finance and Income Inequality: A Review and New Evidence. SSRN Electronic Journal. https://doi.org/10.2139/ssrn.2866399

[14] Deininger, K. y Squire, L. (1998). New ways of looking at old issues: inequality and growth. Journal of Development Economics, 57(2), 259-287. https://doi.org/10.1016/s0304-3878(98)00099-6

[15] Demirgüç-Kunt, A. y Levine, R. (2009). Finance And Inequality: Theory And Evidence. Policy Research Working Papers. https://doi.org/10.3386/w15275

[16] Denk, O. y Cournede, B. (2015). Finance and Income Inequality in OECD Countries. SSRN Electronic Journal. https://doi.org/10.1787/5js04v5jm2hl-en

[17] Dollar, D. y Kraay, A. (2004). Growth is Good for the Poor. Growth, Inequality, and Poverty, 29-61. https://doi.org/10.1093/0199268657.003.0002 
[18] Galor, O. y Zeira, J. (1993). Income Distribution and Macroeconomics. The Review of Economic Studies, 60(1), 35. https://doi.org/10.2307/2297811

[19] Gimet, C. y Lagoarde-Segot, T. (2011). A closer look at financial development and income distribution. Journal of Banking \& Finance, 35(7), 1698-1713. https://doi.org/10.2139/ssrn.1757876

[20] Goodin, R. y Dryzek, J. (1980). Rational Participation: The Politics of Relative Power. British Journal of Political Science, 10(3), 273-292. https://doi.org/10.1017/s0007123400002209

[21] Hamori, S. y Hashiguchi, Y. (2012). The effect of financial deepening on inequality: Some international evidence. Journal of Asian Economics, 23(4), 353-359. https://doi.org/10.1016/j.asieco.2011.12.001

[22] Hsieh, J., Chen, T.-C. y Lin, S.-C. (2019). Financial structure, bank competition and income inequality. The North American Journal of Economics and Finance, 48, 450-466. https://doi.org/10.1016/j.najef.2019.03.006

[23] Jauch, S. y Watzka, S. (2015). Financial development and income inequality: a panel data approach. Empirical Economics, 51(1), 291-314. https://doi.org/10.1007/s00181-015-1008-x

[24] Johansson, A. C. y Wang, X. (2014). Financial sector policies and income inequality. China Economic Review, 31, 367-378. https://doi.org/10.1016/j.chieco.2014.06.002

[25] Kunieda, T., Okada, K. y Shibata, A. (2013). Finance and inequality: how does globalization change their relationship? $\quad$ Macroeconomic 1091-1128 https://doi.org/10.1017/s1365100512000843

[26] Lahoti, R., Jayadev, A. y Reddy, S. (2016). The Global Consumption and Income Project (GCIP): An Overview. Journal of Globalization and Development, 7(1). https://doi.org/10.1515/jgd-2016-0025

[27] Meniago, C. y Asongu, S. A. (2018). Revisiting the finance-inequality nexus in a panel of African countries. Research in International Business and Finance, 46, 399-419. https://doi.org/10.1016/j.ribaf.2018.04.012

[28] Nollert, M., (1995). Neocorporatism and political protest in the western democracies: A cross-national analysis. J.C., Jenkins y B. Klandermans, The Politics of Social Protest: Comparative Perspectives on States and Social Movements (pp. 68-81), London: University College of London Press.

[29] Rajan, R. G. y Zingales, L. (2003). The great reversals: the politics of financial development in the twentieth century. Journal of Financial Economics, 69(1), 5-50. https://doi.org/10.1016/s0304405x(03)00125-9

[30] Ravallion, M. (2004). Growth, Inequality, and Poverty: Looking Beyond Averages. Growth, Inequality, and Poverty, 62-80. https://doi.org/10.1093/0199268657.003.0003

[31] Rice, T. y Strahan, P. E. (2010). Does Credit Competition Affect Small-Firm Finance? The Journal of Finance, 65(3), 861-889. https://doi.org/10.1111/j.1540-6261.2010.01555.x

[32] Schaeck, K., Cihak, M. y Wolfe, S. (2009). Are Competitive Banking Systems More Stable? Journal of Money, Credit and Banking, 41(4), 711-734. https://doi.org/10.1111/j.1538-4616.2009.00228.x

[33] Sehrawat, M., y Giri, A. K. (2015). Financial development and income inequality in India: an application of ARDL approach. International Journal of Social Economics, 42(1), 64-81. https://doi.org/10.1108/ijse-09-2013-0208

[34] Seven, U. y Coskun, Y. (2016). Does financial development reduce income inequality and poverty? Evidence from emerging countries. Emerging Markets Review, 26, 34-63. https://doi.org/10.1016/j.ememar.2016.02.002

[35] Solt, F. (2008). Economic Inequality and Democratic Political Engagement. American Journal of Political Science, 52(1), 48-60. https://doi.org/10.1111/j.1540-5907.2007.00298.x

[36] Solt, F. (2015). Economic Inequality and Nonviolent Protest. Social Science Quarterly, 96(5), 13141327. https://doi.org/10.1111/ssqu.12198

[37] Stiglitz, J.E. (2009). The anatomy of a murder: Who killed America's economy? Critical Review 21, 329339. https://doi.org/10.9783/9780812204933.139 
[38] Thornton, J. y di Tommaso, C. (2020). The long-run relationship between finance and income inequality: Evidence from panel data. Finance Research Letters, 32, 101180. https://doi.org/10.1016/j.frl.2019.04.036

[39] von Ehrlich, M. y Seidel, T. (2015). Regional implications of financial market development: Industry location and income inequality. European Economic Review, 73, 85-102. https://doi.org/10.1016/j.euroecorev.2014.10.008

[40] White, H. y Anderson, E. (2001). Growth versus Distribution: Does the Pattern of Growth Matter? Development Policy Review, 19(3), 267-289. https://doi.org/10.1111/1467-7679.00134

[41] Zhang, R. y Naceur, S. B. (2019). Financial development, inequality, and poverty: Some international evidence. International Review of Economics \& Finance, 61, 1-16. https://doi.org/10.5089/9781498359283.001

\section{Apéndice}

Tabla 1. Países que conforman la muestra del sector bancario

\begin{tabular}{|c|c|c|}
\hline 1. Albania & 26. Georgia & 51. Paraguay \\
\hline 2. $\quad$ Argentina & 27. Alemania & 52. Perú \\
\hline 3. Armenia & 28. Grecia & 53. Filipinas \\
\hline 4. $\quad$ Austria & 29. Honduras & 54. Polonia \\
\hline 5. Bielorrusia & 30. Hungría & 55. Portugal \\
\hline 6. $\quad$ Bélgica & 31. India & 56. Rumania \\
\hline 7. Bolivia & 32. Indonesia & 57. Federación Rusa \\
\hline 8. $\quad$ Brasil & 33. Irlanda & 58. Ruanda \\
\hline 9. Bulgaria & 34. Israel & 59. Senegal \\
\hline 10. Burkina Faso & 35. Italia & 60. Serbia \\
\hline 11. Camboya & 36. Kazajstán & 61. Singapur \\
\hline 12. Camerún & 37. Letonia & 62. Eslovenia \\
\hline 13. Chile & 38. Lituania & 63. Sudáfrica \\
\hline 14. Colombia & 39. Luxemburgo & 64. España \\
\hline 15. Costa Rica & 40. Madagascar & 65. Sri Lanka \\
\hline 16. Croacia & 41. México & 66. Suecia \\
\hline 17. Chipre & 42. Moldavia & 67. Suiza \\
\hline 18. República Checa & 43. Mongolia & 68. Tanzania \\
\hline 19. Dinamarca & 44. Montenegro & 69. Tailandia \\
\hline 20. República Dominicana & 45. Países Bajos & 70. Turquía \\
\hline 21. Ecuador & 46. Nueva Zelanda & 71. Uganda \\
\hline 22. El Salvador & 47. Níger & 72. Ucrania \\
\hline 23. Estonia & 48. Noruega & 73. Estados Unidos \\
\hline 24. Finlandia & 49. Pakistán & 74. Uruguay \\
\hline 25. Francia & 50. Panamá & 75. Vietnam \\
\hline
\end{tabular}

Fuente: Elaboración propia

Tabla 2. Países que conforman la muestra mercados financieros

\begin{tabular}{|l|l|l|}
\hline 1. Argentina, & 20. Hungría & 39. Polonia \\
\hline 2. Austria & 21. India & 40. Portugal \\
\hline 3. Bélgica & 22. Indonesia & 41. Federación Rusa \\
\hline 4. Brasil & 23. Irlanda & 42. Ruanda \\
\hline
\end{tabular}




\begin{tabular}{|l|l|l|}
\hline 5. Bulgaria & 24. Israel & 43. Serbia \\
\hline 6. Chile & 25. Italia & 44. Singapur \\
\hline 7. China & 26. Kazajstán & 45. Eslovenia \\
\hline 8. Colombia & 27. Letonia & 46. Sudáfrica \\
\hline 9. Costa Rica & 28. Lituania & 47. España \\
\hline 10. Croacia & 29. Luxemburgo & 48. Sri Lanka \\
\hline 11. Chipre & 30. México & 49. Suecia \\
\hline 12. República Checa & 31. Mongolia & 50. Suiza \\
\hline 13. Dinamarca & 32. Países Bajos & 51. Tanzania \\
\hline 14. Ecuador & 33. Nueva Zelanda & 52. Tailandia \\
\hline 15. Estonia & 34. Noruega & 53. Turquía \\
\hline 16. Finlandia & 35. Pakistán & 54. Ucrania \\
\hline 17. Francia & 36. Panamá & 55. Reino Unido \\
\hline 18. Alemania & 37. Perú & 56. Estados Unidos \\
\hline 19. Grecia & 38. Filipinas & 57. Vietnam \\
\hline
\end{tabular}

Fuente: Elaboración propia

Tabla 3. Matriz de correlación para la muestra del sector bancario con la variable Des. Atkinson como variable dependiente

\begin{tabular}{|c|c|c|c|c|c|c|c|c|c|c|c|}
\hline & \multicolumn{11}{|c|}{$\mid$ Des. Atki|Estru.CoEstab.cr|E.net_indE.returnEstab.Z_P.Credit|Cont.govCont.Inf|Estru.LeCont.tra } \\
\hline Des.Atkin & 1 & 0.025 & -0.039 & -0.004 & 0.021 & 0.003 & 0.425 & -0.006 & 0.022 & 0.040 & -0.034 \\
\hline Estru.Cor & 0.025 & 1 & -0.055 & -0.085 & 0.016 & -0.021 & -0.004 & 0.021 & -0.050 & -0.102 & -0.042 \\
\hline Estab.cre & -0.039 & -0.055 & 1 & -0.025 & 0.005 & -0.004 & 0.025 & -0.013 & 0.019 & 0.006 & 0.007 \\
\hline E.net_int & -0.004 & -0.085 & -0.025 & 1 & 0.045 & 0.007 & -0.008 & -0.033 & 0.086 & 0.023 & 0.014 \\
\hline E.return & 0.021 & 0.016 & 0.005 & 0.045 & 1 & -0.002 & 0.002 & -0.016 & 0.003 & -0.016 & 0.127 \\
\hline Estab. $Z_{-}$S & 0.003 & -0.021 & -0.004 & 0.007 & -0.002 & 1 & .00007 & -0.012 & -0.003 & 0.024 & 0.014 \\
\hline P.Credit & 0.425 & -0.004 & 0.0256 & -0.008 & 0.002 & .00007 & 1 & -0.014 & 0.025 & -0.001 & 0.012 \\
\hline Cont.gov & -0.006 & 0.021 & -0.013 & -0.033 & -0.016 & -0.012 & -0.014 & 1 & -0.045 & -0.011 & -0.164 \\
\hline Cont. Inf & 0.022 & -0.050 & 0.019 & 0.086 & 0.003 & -0.003 & 0.025 & -0.045 & 1 & -0.008 & 0.132 \\
\hline Estru.Ler & 0.040 & -0.102 & 0.006 & 0.023 & -0.016 & 0.024 & -0.001 & -0.011 & -0.008 & 1 & -0.012 \\
\hline Cont.trad & -0.034 & -0.042 & 0.007 & 0.014 & 0.127 & 0.014 & 0.012 & -0.164 & 0.132 & -0.012 & 1 \\
\hline
\end{tabular}

Fuente: elaboración propia

Tabla 4. Matriz de correlación para la muestra de mercados financieros con la variable Des. Atkinson como variable dependiente

\begin{tabular}{|c|c|c|c|c|c|c|c|c|c|}
\hline & & & We & ok.market & rEst.price_v & E.market_tu & Cont.go & Cont.In & Cont.tro \\
\hline Des. Atkinsor & 1 & 0.160 & 0.019 & 0.004 & 0.010 & -0.004 & 0.020 & 0.046 & -0.038 \\
\hline A.list_comp & 0.160 & 1 & 0.044 & -0.0002 & -0.008 & 0.112 & 0.011 & 0.030 & 0.016 \\
\hline P.market_ca & 0.019 & 0.044 & 1 & 0.031 & -0.048 & 0.003 & -0.050 & -0.0003 & 0.037 \\
\hline R.market_re & 0.004 & -0.0002 & 0.031 & 1 & 0.008 & 0.008 & -0.023 & -0.023 & -0.046 \\
\hline Est.price_vo & 0.010 & -0.008 & -0.048 & 0.008 & 1 & 0.0002 & 0.081 & 0.084 & -0.116 \\
\hline E.market_tu & -0.004 & 0.112 & 0.003 & 0.008 & 0.0002 & 1 & -0.002 & -0.0006 & -0.026 \\
\hline Cont.gov & 0.020 & 0.011 & -0.050 & -0.023 & 0.081 & -0.002 & 1 & -0.076 & -0.210 \\
\hline Cont.Inf & 0.046 & 0.030 & -0.0003 & -0.023 & 0.0845 & -0.0006 & -0.076 & 1 & 0.180 \\
\hline Cont.trade & -0.038 & 0.016 & 0.037 & -0.046 & -0.116 & -0.026 & -0.210 & 0.180 & 1 \\
\hline
\end{tabular}

